\title{
Carbocisteine inhibits the expression of Muc5b in COPD mouse model
}

This article was published in the following Dove Press journal:

Drug Design, Development and Therapy

\author{
Yan Song' \\ Wei Wang ${ }^{2}$ \\ Yanqing Xie' \\ Bin Xiang ${ }^{\prime}$ \\ Xuan Huang' \\ Weijie Guan' \\ Jinping Zheng'
}

'State Key Laboratory of Respiratory Disease, National Clinical Research Center for Respiratory Disease, Guangzhou Institute of Respiratory Health, First Affiliated Hospital of Guangzhou Medical University, Guangzhou 510120, People's Republic of China; ${ }^{2}$ Drug Research Institute of Guangzhou BaiYunShan Pharmaceutical General Factory, Guangzhou, 5105I5, People's Republic of China
Correspondence: Jinping Zheng First Affiliated Hospital of Guangzhou Medical University, No I5I Yanjiang Road, Guangzhou 510120, People's Republic of China

Tel +86 2083062869

Fax +86 2083062729

Email jpzhenggy@163.com
Background: Cigarette smoke (CS) results in chronic mucus hypersecretion and airway inflammation, contributing to COPD pathogenesis. Mucin 5B (MUC5B) and mucin 5 AC (MUC5AC) are major mucins implicated in COPD pathogenesis. Carbocisteine can reduce mucus viscosity and elasticity. Although carbocisteine decreased human elastase-induced MUC5AC expression in vitro and reduced MUC5AC expression that alleviated bacteria adhesion and improved mucus clearance in vivo, the roles of carbocisteine in inducing MUC5B expression in COPD remain unclear.

Methods: To investigate the Muc5b/Muc5ac ratio and the gene and protein levels of Muc5b in COPD and carbocisteine intervention models. C57B6J mice were used to develop COPD model by instilling intratracheally with lipopolysaccharide on days 1 and 14 and were exposed to CS for $2 \mathrm{hr}$ twice a day for 12 weeks. Low and high doses of carbocisteine 112.5 and $225 \mathrm{mg} / \mathrm{kg} / \mathrm{d}$, respectively, given by gavage administration were applied for the treatment in COPD models for the same duration, and carboxymethylcellulose was used as control. Carbocisteine significantly attenuated inflammation in bronchoalveolar lavage fluid and pulmonary tissue, improved pulmonary function and protected against emphysema.

Results: High-dose carbocisteine significantly decreased the overproduction of Muc5b $(P<0.01)$ and Muc5ac $(P<0.001)$, and restored Muc5b/Muc5ac ratio in COPD model group $(P<0.001)$. Moreover, the Muc5b/Muc5ac ratio negatively correlated with pro-inflammatory cytokines such as IL-6 and keratinocyte-derived cytokine, mean linear intercept, functional residual capacity and airway resistance, but positively correlated with dynamic compliance. Conclusions: These findings suggest that carbocisteine attenuated Muc5b and Muc5ac secretion and restored Muc5b protein levels, which may improve mucus clearance in COPD. Keywords: COPD, cigarette smoke, Muc5b, Muc5ac, carbocisteine

\section{Introduction}

COPD is a chronic airway disease characterized by persistent respiratory symptoms (such as mucus hypersecretion) and airflow limitation mostly associated with cigarette smoke (CS). CS-induced chronic mucus hypersecretion (CMH) and airway inflammation are critical targets contributing to COPD pathogenesis. ${ }^{1}$ Importantly, $\mathrm{CMH}$ is associated with airway inflammation ${ }^{2}$ and lung function decline in COPD. ${ }^{3}$

Medications which decrease $\mathrm{CMH}$ may confer therapeutic effects on the treatment of COPD. Carbocisteine, a well-tolerated mucolytic agent, has been extensively used in COPD. ${ }^{4,5}$ Carbocisteine has been shown to markedly prevent COPD exacerbations ${ }^{6}$ and alleviate oxidative stress and inflammatory-induced injury in vitro ${ }^{7-9}$ and in vivo. $^{10,11}$ Carbocisteine also altered mucus properties by balancing the fucose and 
sialic acid contents on mucins. ${ }^{12,13}$ Mucin 5B (MUC5B) and mucin 5AC (MUC5AC) are major secreted mucins highly expressed in the airways and are implicated in respiratory diseases with $\mathrm{CMH} .{ }^{14,15}$ MUC5AC is responsible for airway hyper-responsiveness in asthma mice model ${ }^{16}$ and increased significantly in patients with asthma. ${ }^{17}$ Unlike MUC5AC, production of MUC5B is decreased in asthma. ${ }^{18}$ Roy et al found that Muc5b was critical to maintaining airway defense. ${ }^{19}$ Moreover, MUC5B production was significantly augmented and the ratio of MUC5B to MUC5AC (MUC5B/ MUC5AC ratio) was increased and correlated with lower FEV1 in COPD. ${ }^{20}$ Therefore, changes in mucin components might play important roles in COPD pathogenesis.

Although carbocisteine decreased human elastaseinduced MUC5AC expression in NCI-H292 cells ${ }^{21}$ and reduced MUC5AC expression that alleviated bacteria adhesion and improved mucus clearance, ${ }^{6}$ the roles of carbocisteine in inducing Muc5b expression in COPD remain unclear.

Importantly, we have previously proved that carbocisteine markedly reduced the rate of COPD acute exacerbations ${ }^{6}$ and alleviated oxidative stress and inflammatory-induced injury, which have been shown to activate MAPK and NF-kB signaling pathways, ${ }^{7,8}$ reinforcing the possibility that carbocisteine may regulate Muc5b expression via activation of MAPK and NF-kB signaling pathways in COPD. We sought to investigate whether carbocisteine confers therapeutic effects through altering Muc5ac and Muc5b expression in COPD mice model.

\section{Materials and methods}

\section{Preparation of carbocisteine and} carboxymethylcellulose

Carbocisteine (Wuhan Yuanda Hong Yuan Pharmaceutical Company, Wuhan, China) was dissolved in $0.3 \%$ carboxymethylcellulose solution for homogenization. Mice were administered with a gavage of carbocisteine at $112.5 \mathrm{mg} /$ $\mathrm{kg} / \mathrm{d}$ or $225 \mathrm{mg} / \mathrm{kg} / \mathrm{d}$ for 12 weeks, respectively.

\section{Establishment of COPD mice model}

Cigarette (Guangdong tobacco industry, Guangzhou, China) smoking exposure combined with airway lipopolysaccharide (LPS) inhalation could imitate the occurrence and development of COPD. ${ }^{22,23}$ Male C57B6J mice at 8 weeks of age $(21.6 \pm 1.5 \mathrm{~g})$ were provided by Nanjing Institute of Biomedical Sciences, and were randomly divided into four groups ( $\mathrm{n}=12$ each): control [normal saline (NS) intratracheal instillation], COPD (CS exposure with LPS intratracheal instillation), low-dose carbocisteine group (CS exposure with LPS intratracheal instillation +112.5 $\mathrm{mg} / \mathrm{kg} / \mathrm{d}$ carbocisteine), high-dose carbocisteine group (CS exposure with LPS intratracheal instillation $+225 \mathrm{mg} / \mathrm{kg} / \mathrm{d}$ carbocisteine). LPS or NS was instilled intratracheally at $7.5 \mu \mathrm{g} / 50 \mu \mathrm{L}$ under anesthesia with isoflurane at days 1 and 14 (Figure 1A). Mice were exposed to CS or room air for 2 hrs twice daily for 12 weeks. Furthermore, carboxymethylcellulose and different doses of carbocisteine were administered orally once daily to mice for 12 weeks (Figure 1B). Water and diet were freely accessed. All animal experiments were approved by the Animal Ethical Committee of Guangzhou Medical University and according to the National Institutes Guidelines.

\section{Lung function measurement}

At week 12, pulmonary function was measurement in a forced pulmonary maneuver system (Buxco Research Systems, USA). Animals were mechanically ventilated and the functional residual capacity (FRC), airway resistance (RI) and dynamic compliance (Cdyn) were recorded, as previously described. ${ }^{1}$

\section{Bronchoalveolar lavage (BALF) and lung tissue sections}

BALF was obtained via tracheal instillation thrice of ice-cold saline $(0.6 \mathrm{~mL})$, with a mean of $1.5 \mathrm{~mL}$ fluid recovered. BALF was used to determine protein concentrations with ELISA and inflammatory cell count was assessed under light microscopy. The left lung was fixed with $10 \%$ formalin at a constant hydrostatic pressure of $25 \mathrm{~cm} \mathrm{H}_{2} \mathrm{O} .^{24}$ The sample was then dehydrated and embedded in paraffin, followed by staining of tissue sections with HE for assessing histopathology by calculating the mean linear intercept (MLI). The right lung was homogenized immediately with Trizol reagent (Invitrogen, USA) and stored at $-80^{\circ} \mathrm{C}$ for subsequent qPCR assessment. ${ }^{25}$

\section{ELISA}

We measured Muc5ac and Muc5b (Santa Cruz, USA) protein levels with ELISA, as previously described. ${ }^{12,26}$ The concentration of IL-6 (eBioscience, USA) and keratinocyte-derived cytokine (KC) (R\&D Systems Inc.,USA) were determined with ELISA kits following the manufacturers' protocol. 
$\mathbf{A}$
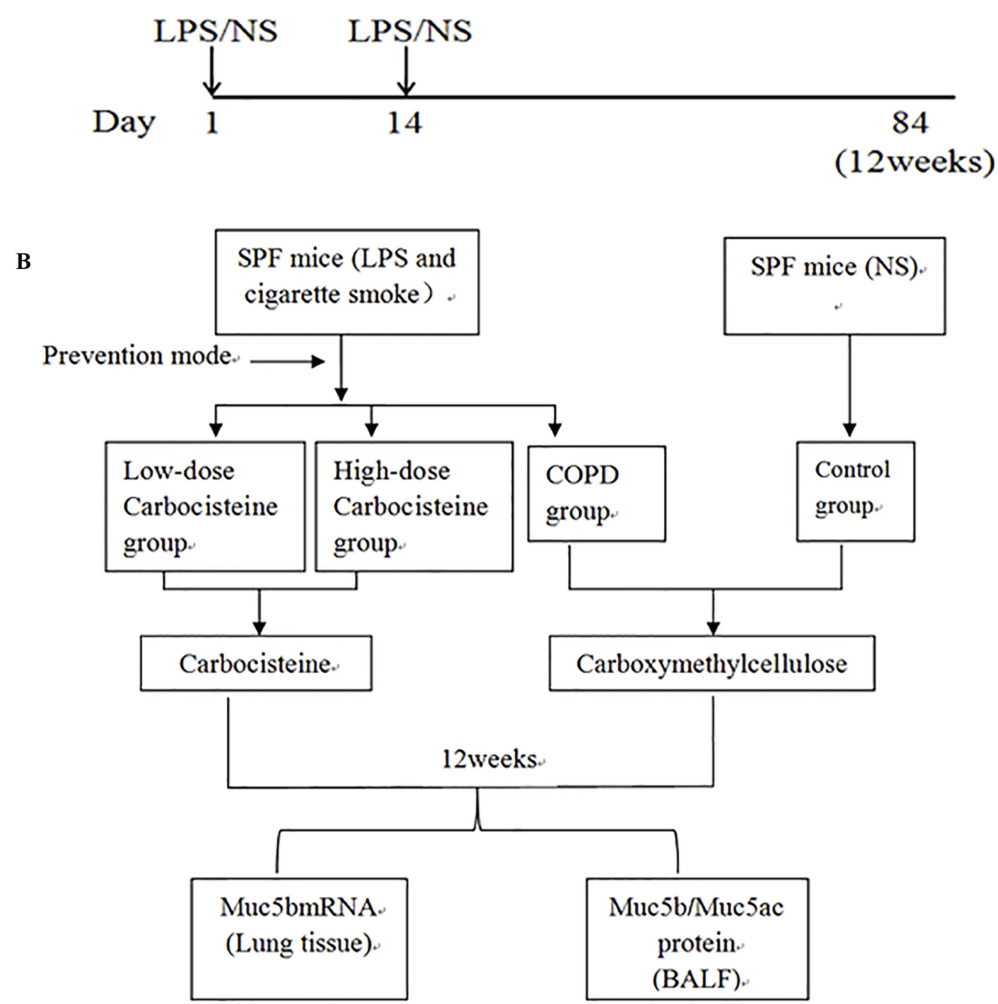

Figure I (A) Protocol for LPS or NS intratracheal instillation. (B) The technical roadmap of this study.

\section{RNA extraction and real-time PCR}

Total RNA was extracted and synthesized cDNA from lung tissue homogenate, ${ }^{25}$ and $1 \mathrm{mg}$ of total RNA was reverse transcribed using the Prime Script RT Reagent Kit (Perfect Real Time; Takara, Japan) to detect relative mRNAs. Quantitative real-time PCR was performed in triplicates on a LightCycler $^{\circledR} 480$ II quantitative PCR system (Roche, Applied Science) as previously described..$^{27}$ The cycle conditions were as follows: $95^{\circ} \mathrm{C}$ for 15 mins followed by $94^{\circ} \mathrm{C}$ for $5 \mathrm{~s}, 40$ cycles of $57.5^{\circ} \mathrm{C}$ for $15 \mathrm{~s}, 72^{\circ} \mathrm{C}$ for $20 \mathrm{~s}$ and $95^{\circ} \mathrm{C}$ for $1 \mathrm{~min}$. The $\mathrm{Ct}$ values obtained from different samples were compared using the $2^{-\Delta \Delta \mathrm{Ct}}$ method. Primers of genes were designed by Sangon Biotech (Shanghai, China). The primer sequences were as follows: Muc $5 b^{28}$ Forward: 5'- GTGAGGAGGACT CCTGTCAAGT-3' and Reverse: 5'-CCTCGCAGAAGGTG ATGTTG-3'; Muc5ac ${ }^{16}$ Forward:5'- ATGGGCTGTGTTCCT GTGTC- $3^{\prime}$ and Reverse: $5^{\prime}$ - CAGAACATGTGTTGGTGCA GTC-3'; tumor necrosis factor- $\alpha$ (TNF- $\alpha)^{29}$ Forward: $5^{\prime}$-AGG GTCTGGGCCATAGAACT- ${ }^{\prime}$ and Reverse: $5^{\prime}$-CCA CCAC GCTCTTCTGTCTAC-3'; $18 \mathrm{~S}^{30}$ Forward: 5'-GCAATTATTC CCCATGAA CG-3' and Reverse: 5'-GGCCTCACTAAACC ATCCAA-3'; GAPDH ${ }^{31}$ Forward: 5'- GGTGAAGGTCGG TGTGAACG-3' and Reverse: 5'-CTCGCTCCTGGAAGA
TGGTG-3'. The relative gene expression levels were normalized to the endogenous control (18S and GAPDH). The $18 \mathrm{~S}$ and GAPDH are one of the most commonly used housekeeping genes used in comparisons of gene expression data. ${ }^{11,28,43}$

\section{Statistical analysis}

Data were expressed as mean $\pm \mathrm{SD}$ or medians (inter-quartile range). Comparisons of multiple groups were analyzed using one-way ANOVA or nonparametric test if appropriate. The correlations between Muc5b/Muc5ac ratio and other indices were assessed with Spearman or Pearson correlation coefficient. $P<0.05$ was considered statistically significant. All analyses were performed using SPSS version 22.0 (SPSS Inc., USA).

\section{Results}

\section{Successfully established COPD mice model}

CS exposure with LPS intratracheal instillation established COPD mice model which exhibited emphysematous destruction, remodeling of airway epithelial, chronic mucus hypersecretion and increased inflammatory cells in the BALF and lung tissue, compared with the control 
group. Furthermore, pulmonary function in COPD group was decreased, which manifested a decrease in Cdyn and increases in RI and FRC. All these results indicated that the model we established was available for pathophysiological studies of COPD.

\section{Carbocisteine attenuated Muc5ac and Muc5b overproduction in COPD mice model}

We noted significant increases in Muc5ac and Muc5b protein levels in COPD mice models. Carbocisteine, especially at higher doses, significantly reduced Muc5ac and Muc5b in BALF (Figure 2A and B). We also found substantially lower Muc5b/Muc5ac ratio in COPD mice models compared with control group $(1.6 \pm 0.8$ vs $3.8 \pm 1.5 ; P<0.01)$. The Muc5b/ Muc5ac ratio was higher in carbocisteine treatment groups, especially in the high-dose group $(P<0.001$, Figure $2 \mathrm{C})$. Muc5b and Muc5ac genes were expressed at high levels in COPD mice models (Figure 2D and E). However, high-dose carbocisteine attenuated Muc $5 \mathrm{~b}$ gene expression significantly $(P<0.01$, Figure 2D) and decreased Muc5ac gene expression levels (Figure 2E).

\section{Effects of carbocisteine on lung tissue inflammation}

In COPD mice model, we observed infiltration of inflammatory cells, predominantly neutrophils, in the peribronchial and alveolar regions. Administration with carbocisteine, especially at higher doses, attenuated inflammatory cells infiltration (Figure 3A). Similar findings were observed for the total BALF inflammatory cell count, particularly in the high-dose carbocisteine group (Figure 3B). Consistently, BALF pro-inflammation cytokines such as KC and IL-6 were increased in COPD mice models, which tended to be reduced in high-dose carbocisteine group (Figure 3C and D). We also found that high-dose carbocisteine significantly inhibited TNF- $\alpha$ expression in lung tissues (Figure 3E).

\section{Carbocisteine protected against emphysema in COPD mice model}

HE staining of lung tissues in COPD mice model revealed destructed alveolar walls, with significantly enlarged alveolar spaces and inflammatory cell infiltration (Figure 4A). In contrast, in the control group, the alveolar walls remained intact without inflammatory cell infiltration. The MLI significantly increased by 1.7 -fold in COPD mice model compared with the control group, whereas carbocisteine significantly reduced the MLI from $68.6 \pm 2.6 \mu \mathrm{m}$ to 57.5 $\pm 5.3 \mu \mathrm{m}(P<0.001)$ in low-dose carbocisteine group and from $68.6 \pm 2.6 \mu \mathrm{m}$ to $49.4 \pm 4.0 \mu \mathrm{m}(P<0.001)$ in high-dose carbocisteine group. The MLI differed significantly between the two carbocisteine groups $(P<0.01)$ (Figure 4B).

\section{Effects of carbocisteine on pulmonary function}

There were significant increases in FRC $(0.6 \pm 0.13$ vs 0.39 $\pm 0.10, P<0.01)$ and RI $(0.79 \pm 0.12$ vs $0.55 \pm 0.10, P<0.001)$ and decreases in Cdyn $(0.02 \pm 0.006$ vs $0.03 \pm 0.005$, $P<0.01)$ in COPD mice model, compared with control group (Figure 5). However, FRC remained unchanged throughout treatment courses (Figure 5A). In the lowand high-dose carbocisteine groups, there were significant improvements in RI $(P<0.01, P<0.001$, respectively) and Cdyn $(P<0.05, P<0.01$, respectively), compared with COPD model group (Figure $5 \mathrm{~B}$ and $\mathrm{C}$ ), but all the pulmonary function parameters were no significant difference between the two carbocisteine treatment groups.

\section{Correlations between the Muc5b/Muc5ac ratio and other parameters}

The correlations between the Muc5b/Muc5ac ratio and lung function, MLI, IL-6, KC and TNF- $\alpha$ mRNA levels were examined in all experimental mice. Overall, we found an inverse correlation between the Muc5b/Muc5ac ratio and other parameters, including the FRC, RI, MLI, total cell count, IL-6, KC and TNF- $\alpha$ mRNA. However, the Muc5b/Muc5ac ratio positively correlated with Cdyn in all experimental mice model (Table 1).

\section{Discussion}

We found that carbocisteine significantly restored the Muc5b/Muc5ac ratio, together with decreased neutrophil counts, KC and IL- 6 levels, and TNF- $\alpha$ mRNA expression in COPD mice model. Furthermore, carbocisteine significantly improved lung function, as reflected by the RI and Cdyn. Notably, the Muc5b/Muc5ac ratio negatively correlated with pro-inflammatory cytokines levels, FRC and RI, but positively correlated with Cdyn. Carbocisteine also dose-dependently attenuated airway inflammation and reduced MLI and expression of Muc5b.

In addition to attenuating $\mathrm{CMH}$, carbocisteine has been shown to alleviate inflammatory cell infiltration and decrease pro-inflammation cytokines levels in COPD 


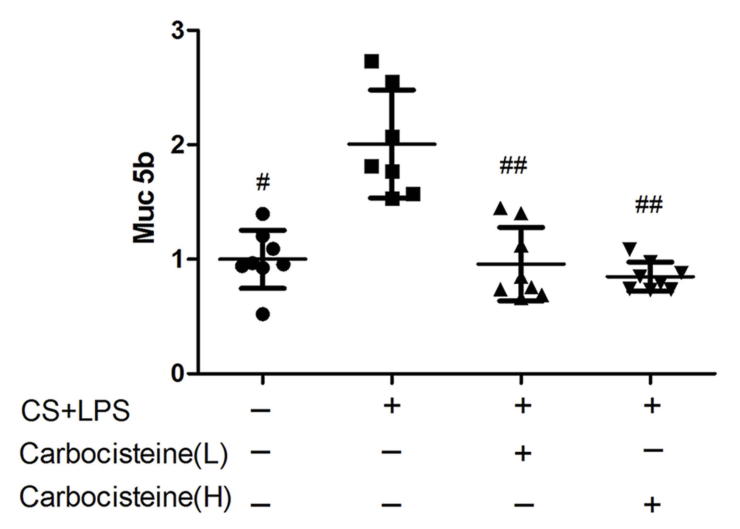

C

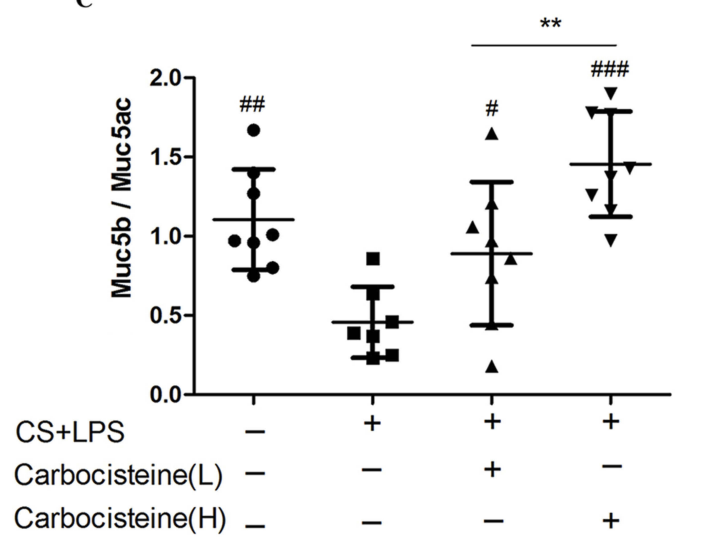

$\mathbf{E}$

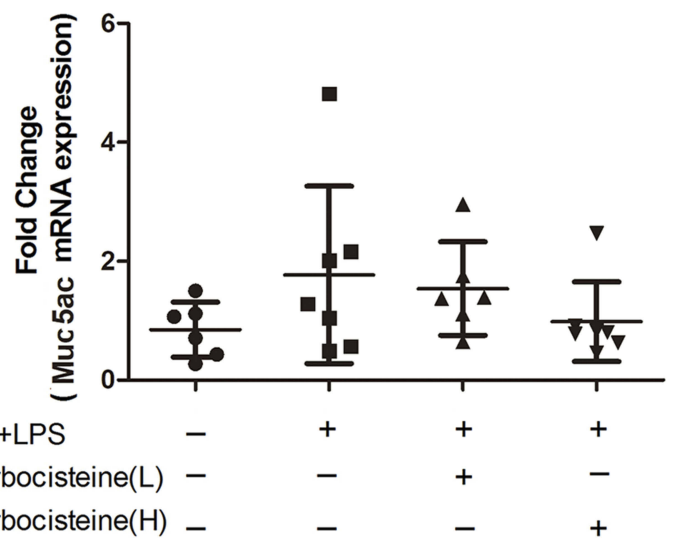

B

CS+LPS

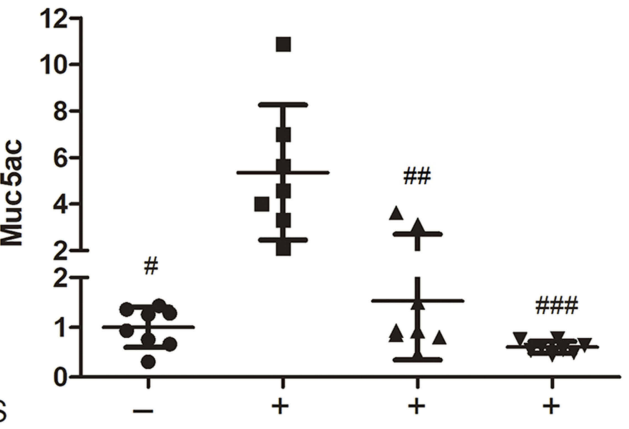

Carbocisteine $(\mathrm{L})-\quad+\quad+\quad-$

Carbocisteine $(\mathrm{H})$ - $\quad-\quad \quad-\quad+$

D

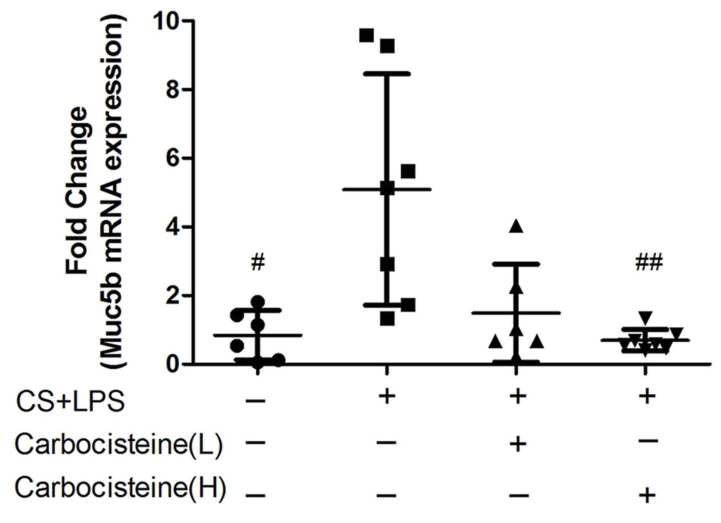

Figure 2 (A) The levels of Muc5b protein in BALF. (B) The levels of Muc5ac protein in BALF. Data are expressed as medians. (A nonparametric Wilcoxon rank-sum test was applied for comparisons between study groups). (C) The Muc5b/Muc5ac ratio in BALF; (D) Muc5b, (E) Muc5ac gene expression is normalized against I8S expression. Data are expressed as mean \pm SD. $n=7-1$ I. (One-way ANOVA with LSD multiple comparisons test) $\# P<0.05$, \#\#P<0.0I, \#\#P<0.00I compared with COPD mice model, $* * P<0.01$ compared between carbocisteine groups.

mice models. ${ }^{10,11,25}$ We sought to investigate whether carbocisteine, especially when administered at higher doses, could significantly attenuate pro-inflammatory cytokines. Our findings indicated that carbocisteine conferred a dosedependent therapeutic effect, which was in agreement with our previous findings related to the anti-inflammatory actions of carbocisteine in vitro. ${ }^{7,8}$ However, Hanaoka et al showed no significant differences between the two carbocisteine treatment groups. ${ }^{10}$ Several explanations were likely to be responsible for such a difference. First, the parameters we measured were mucins and pro-inflammation cytokines which were not the same. Additionally, 

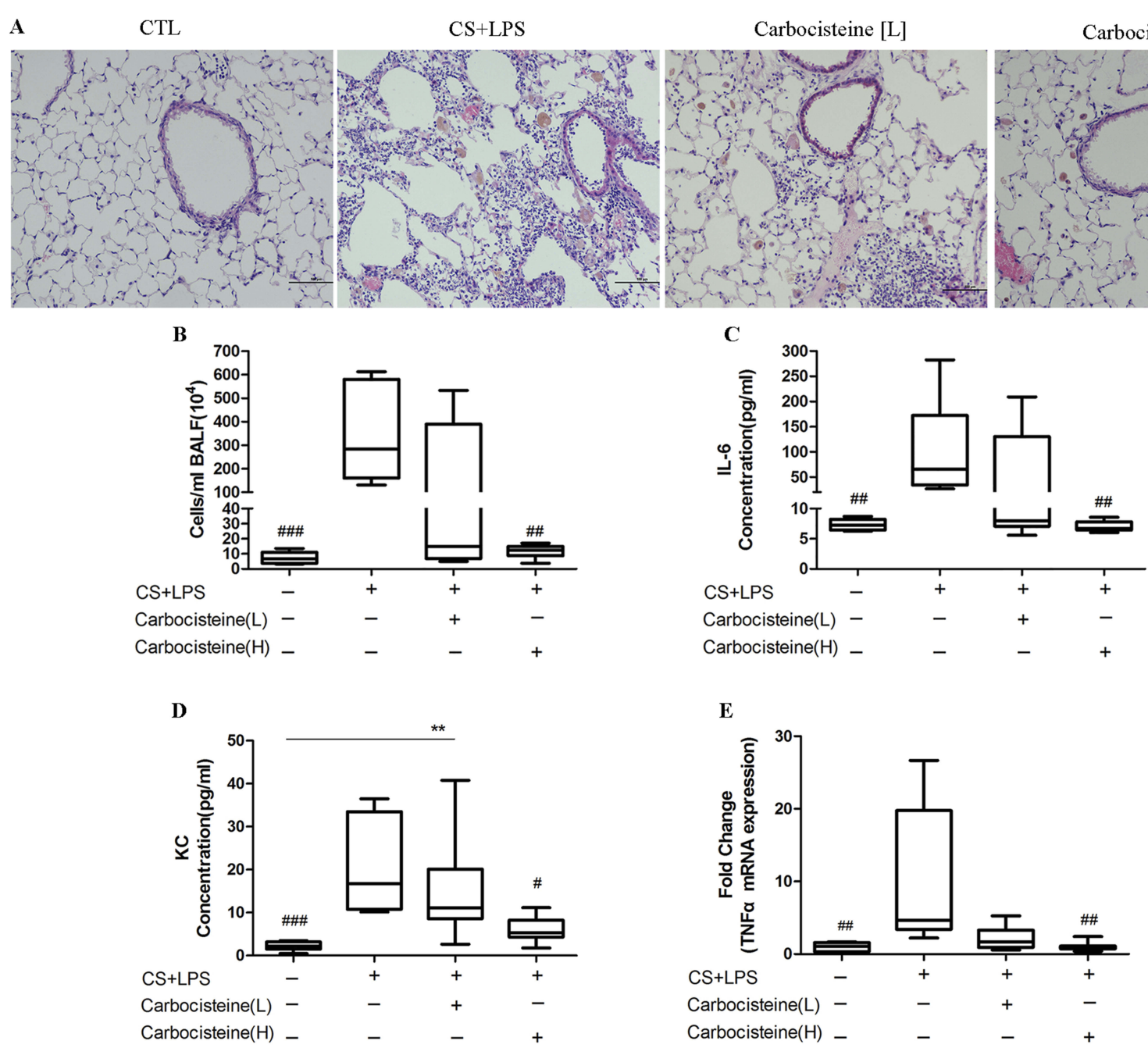

Figure 3 Effects of carbocisteine on lung inflammation in COPD mice model. (A) Pulmonary pathology in four groups. Scale bars, $100 \mu \mathrm{m}$. (B) The total inflammatory cell counts and the concentrations of pro-inflammatory cytokines (IL-6 (C) and KC (D)) in BALF. (E) The pro-inflammatory cytokines (TNF- $\alpha$ ) in lung tissue are measured by qPCR. Data are expressed as medians (inter-quartile range), $n=8-1 \mathrm{I}$. \#P<0.05, \#P<0.0I, \#\#P<0.00I compared with COPD mice models (a nonparametric Wilcoxon ranksum test was applied for comparisons among multiple groups).

carbocisteine was administered at different doses. In our study, $225 \mathrm{mg} / \mathrm{kg} / \mathrm{d}$ carbocisteine in mice was equivalent to $1500 \mathrm{mg} / \mathrm{d}$ in COPD patients. Our previous study has shown that $1500 \mathrm{mg} / \mathrm{d}$ carbocisteine was sufficient to markedly reduce the risk of COPD exacerbations. ${ }^{6}$ Third, the duration of administration with carbocisteine was 12 weeks which was much longer than 21 days in the study by Hanaoka et al.

$\mathrm{CMH}$ is the key element leading to small-airway airflow obstruction, remodeling of airway epithelial and lung function decline. ${ }^{1,3}$ Roy and colleagues showed that Muc5b, but not Muc5ac, was required for airway defense and that the decrease of Muc5b could hamper mucus clearance. ${ }^{19}$ Additionally, publications have stated that the levels of MUC5B in BALF and sputum were increased in COPD patients, ${ }^{32-34}$ while carbocisteine could balance fucosylated and sialylated sugar chains, ${ }^{13}$ normalize the viscous property of mucus ${ }^{12}$ and reduce MUC5AC expression. We speculated that the decrease of Muc5b might have played an important role in COPD pathogenesis and carbocisteine might reduce the expression of Muc5b in COPD mice model. Our data supported this hypothesis by showing that carbocisteine reduced Muc $5 \mathrm{~b}$ expression and restored the Muc5b/Muc5ac ratio in a dose-dependent manner in COPD mice model. The decrease of the Muc5b/ Muc5ac ratio in COPD mice model was in contrast to the findings in COPD patients reported in Kokam et al's study, ${ }^{20}$ in which COPD patients without acute exacerbations within 6 weeks were observed, whereas in our COPD mice model, inflammatory cells infiltration was found and 
A

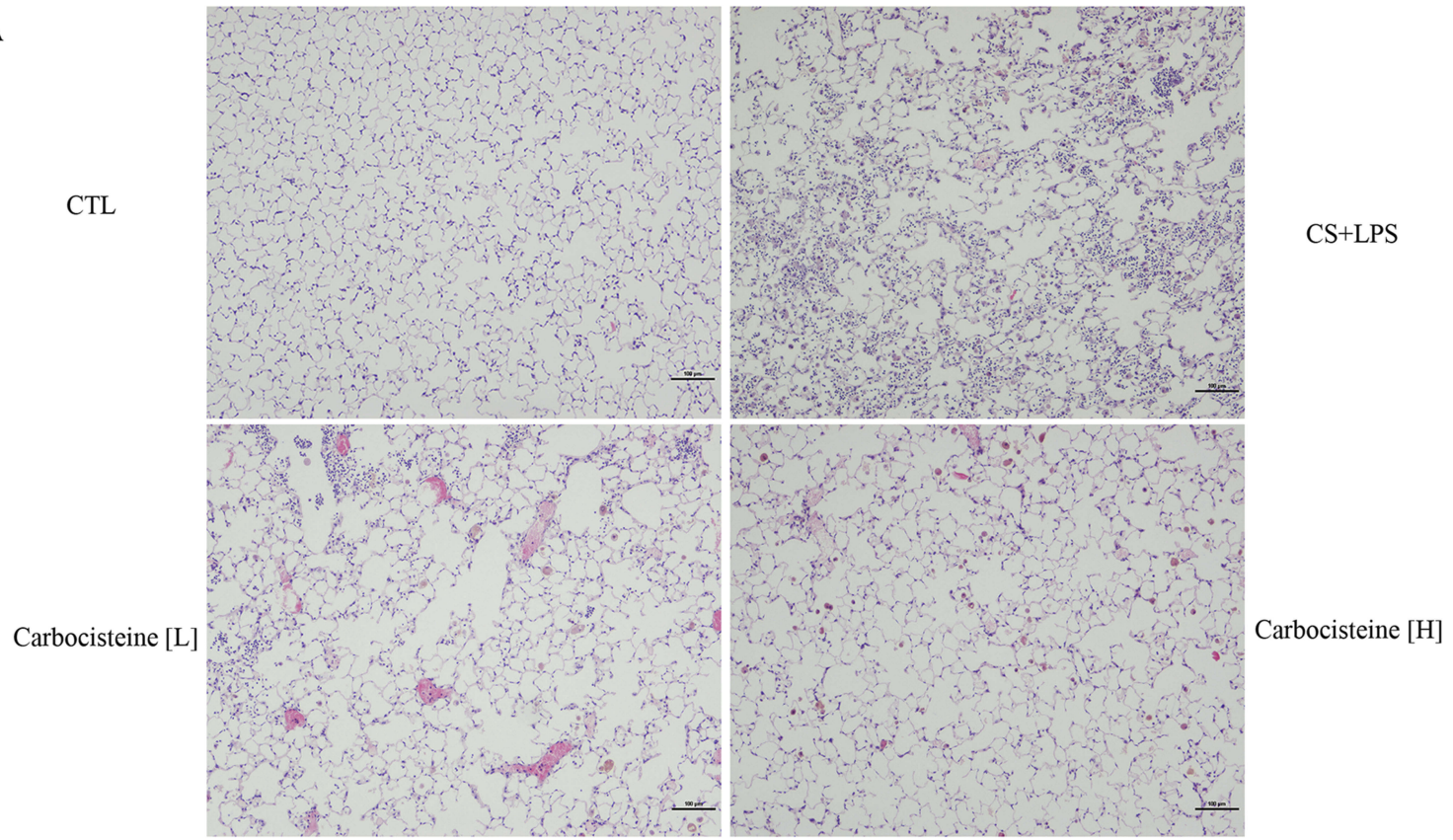

B

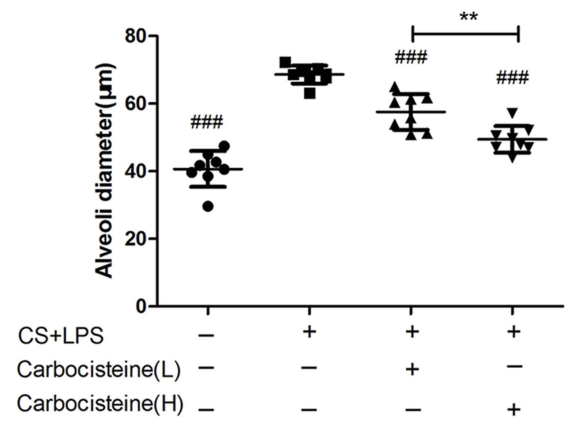

Figure 4 (A) Alveolar architecture of four groups stained with HE staining. Scale bars, $100 \mu \mathrm{m}$. (B) Morphometric measurements of MLI in lung tissues. Data are expressed as mean $\pm S D, n=8$. \#\# $<0.00$ I compared to model group, $* * P<0.01$ compared between the two carbocisteine groups (one-way Analysis of Variance (ANOVA) with Fisher's Least Significant Difference (LSD) multiple comparisons test).

inflammatory mediators closely correlated with the excessive secretion of Mucin. Moreover, mucin we measured in BALF which may be different from that in induced sputum.

To our knowledge, our data were the first to investigate the Muc5b/Muc5ac ratio in carbocisteine prevention of COPD mice model. We specifically showed that the increased ratio of Muc5b over Muc5ac correlated with increased pro-inflammatory cytokines levels and higher pulmonary parameters such as RI and FRC and lower Cdyn. This increased Muc5b/Muc5ac ratio (the relative decrease in Muc5b) may alter mucin composition and attenuate mucus clearance. ${ }^{19}$ The unbalance of the Muc5b/ Muc5ac ratio likely represents a major cause of mucus plugging in COPD pathogenesis. Our previous studies showed that carbocisteine attenuated hydrogen peroxide and TNF-alpha-induced inflammation in vitro via suppressing NF-kB and ERK1/2 MAPK signaling pathways. ${ }^{7,8}$ Additionally, IL-6 and IL-17 regulate Muc5b expression via the ERK signaling pathway, ${ }^{35}$ and IL-1b and IL-17A mediate Muc5b expression by NF-kB-based transcriptional mechanism in vitro. ${ }^{28}$ We hypothesized that carbocisteine may regulate Muc5b expression via suppressing NF-kB and ERK1/2 MAPK signaling pathways in COPD mice model, thereby affecting the progression of COPD. Further studies are needed to explore the signaling pathways of carbocisteine that may impact COPD progression.

CS-induced model has been a well-established method for studying COPD in mice. The CS-induced model may present with hallmark features mimicking human diseases, ${ }^{36-38}$ which is particularly true that CS exposure plus airway LPS inhalation for short-term studies. ${ }^{22,39}$ In our study, mice were instilled with LPS intratracheally 
A

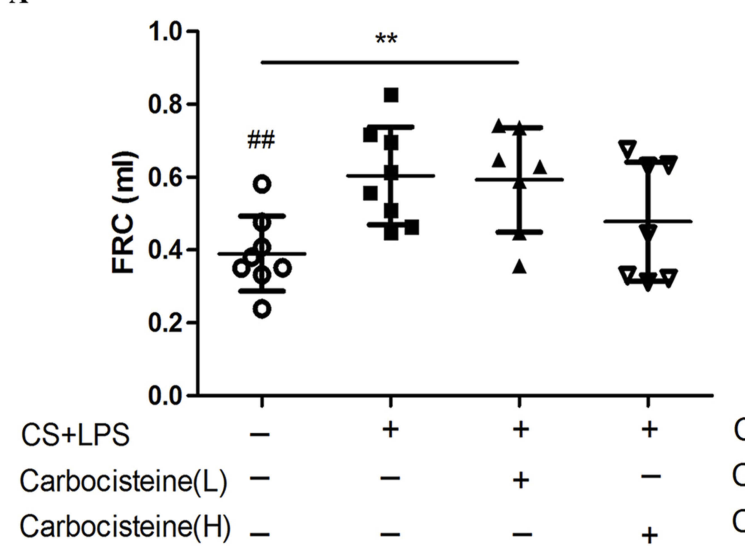

B

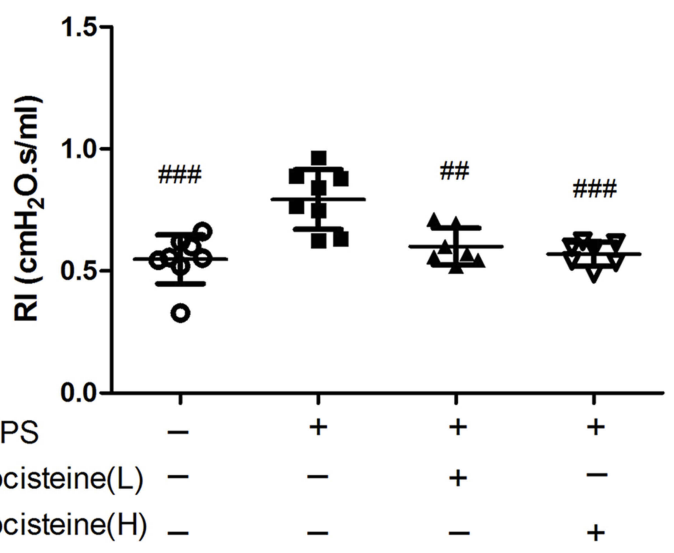

C

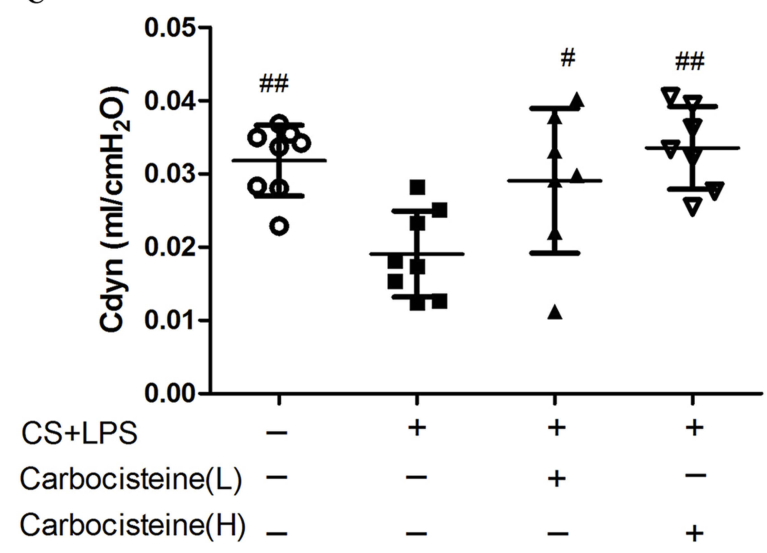

Figure 5 Effects of treatment with carbocisteine on lung function impairment in COPD mice model. (A) Functional residual capacity (FRC), (B) airway resistance (RI), (C) dynamic compliance (Cdyn). This was separately calculated for each group. Data are expressed as mean $\pm S D, n=7-8$. \#P<0.05, \#P<0.01, \#\#P<0.001 compared to model group; $* * P<0.0$ l between the underlined groups. (one-way ANOVA with LSD multiple comparisons test).

Table I Correlation between the Muc5b/Muc5ac ratio and other parameters

\begin{tabular}{|l|l|l|l|}
\hline \multirow{2}{*}{} & \multicolumn{3}{|l|}{ The Muc5b/Muc5ac ratio } \\
\cline { 2 - 4 } & N & $P$ & $P$ \\
\hline FRC & 29 & -0.442 & $<0.05$ \\
RI & 29 & -0.439 & $<0.05$ \\
Cdyn & 29 & $0.56 I$ & $<0.01$ \\
MLI & $3 \mathrm{I}$ & -0.506 & $<0.01$ \\
Total cells & $3 \mathrm{I}$ & -0.432 & $<0.05$ \\
IL-6 & $3 \mathrm{I}$ & $-0.6 \mathrm{II}$ & $<0.001$ \\
KC & $3 \mathrm{I}$ & -0.540 & $<0.01$ \\
TNF- $\alpha$ mRNA & $3 \mathrm{I}$ & -0.420 & $<0.05$ \\
\hline
\end{tabular}

instead of intranasally, in order to ensure the precise administration of LPS at the target location, which should be regarded as one of the most innovative aspects of our study. Moreover, although many studies have shown an increase in MUC5AC expression in COPD patients or smokers $^{40-42}$ and that carbocisteine could reduce the overproduction of MUC5AC, ${ }^{11,25}$ no study so far has investigated how carbocisteine influence on MUC5B expression. Our study was the first to provide scientific observation on the mode of action of carbocisteine on Muc5b expression induced by CS exposure with LPS intratracheally instillation in a dose-dependent manner in COPD mice model.

Some limitations should be mentioned. First, we did not measure the specific concentration of Muc5ac or Muc5b. However, there were no widely accepted ELISA kits or standards for detection of Muc5ac and Muc5b in BALF of mice. Furthermore, the methods we used were in accordance with those in Yageta et al's study. ${ }^{11}$ Second, we did not use immunohistochemistry for analyzing mucin localization. Muc5b was produced mainly in cells of submucosal glands. ${ }^{15}$ However, there were few glands in lung pathology in our study. The next step is to sample the trachea of mice to further analyze the mucin localization in the treatment group. In addition, Muc $5 b^{-/-}$mice should 
be used to establish COPD model to further determine how carbocisteine impact on Muc5b expression.

In conclusion, carbocisteine decreased the secretion of Muc5ac and Muc5b and restored Muc5b protein levels, thereby improving lung function and alleviating the inflammatory responses, which may improve mucus clearance and provide a more in-depth explanation of the pharmacological actions for clinical application of mucolytics, such as carbocisteine, in patients with COPD.

\section{Acknowledgment}

This study was supported by grants from the National Key R\&D Program of China (No.2018YFC1311900), the National Natural Science Foundation of China (No.81470234), the Guangdong provincial project for great new drug research and development (No. 2012A080201003) and the National Key Technology R\&D Program (No.2012BAI05B01, 2013BAI09B09).

\section{Author contributions}

All authors contributed to data analysis, drafting or revising the article, gave final approval of the version to be published, and agree to be accountable for all aspects of the work.

\section{Disclosure}

The abstract has been submitted to the 22nd Congress of the Asian Pacific Society of Respirology, International Convention Centre, Sydney, Australia, 23-26 November 2017 and has been published in Respirology, Vol 22 Issue S3. The authors report no other conflicts of interest in this work.

\section{Abbreviations}

$\mathrm{CS}$, cigarette smoke; BALF, bronchoalveolar lavage fluid; $\mathrm{KC}$, keratinocyte-derived cytokine; MLI, mean linear intercept; $\mathrm{CMH}$, chronic mucus hypersecretion; LPS, Lipopolysaccharide; FRC, The functional residual capacity; RI, airway resistance; Cdyn, dynamic compliance.

\section{References}

1. Vanoirbeek JA, Rinaldi M, De Vooght V, et al. Noninvasive and invasive pulmonary function in mouse models of obstructive and restrictive respiratory diseases. Am J Respir Cell Mol Biol. 2010;42:96-104. doi:10.1165/rcmb.2008-0487OC

2. Maestrelli P, Saetta M, Mapp CE, Fabbri LM. Remodeling in response to infection and injury. Airway inflammation and hypersecretion of mucus in smoking subjects with chronic obstructive pulmonary disease. Am J Respir Crit Care Med. 2001;164:S76-S80. doi:10.1164/ ajrccm.164.supplement_2.2106067
3. Allinson JP, Hardy R, Donaldson GC, et al. The presence of chronic mucus hypersecretion across adult life in relation to chronic obstructive pulmonary disease development. Am J Respir Crit Care Med. 2016;193:662-672. doi:10.1164/rccm.201511-2210OC

4. Tatsumi K, Fukuchi Y, Group PS. Carbocisteine improves quality of life in patients with chronic obstructive pulmonary disease. J Am Geriatr Soc. 2007;55:1884-1886. doi:10.1111/j.1532-5415.2007.01373.x

5. Yasuda H, Yamaya M, Sasaki T, et al. Carbocisteine reduces frequency of common colds and exacerbations in patients with chronic obstructive pulmonary disease. J Am Geriatr Soc. 2006;54:378-380. doi:10.1111/j.1532-5415.2005.00592 9.x

6. Zheng JP, Kang J, Huang SG, et al. Effect of carbocisteine on acute exacerbation of chronic obstructive pulmonary disease (PEACE Study): a randomised placebo-controlled study. Lancet. 2008;371:2013-2018. doi:10.1016/S0140-6736(08)60869-7

7. Wang W, Guan WJ, Huang RQ, et al. Carbocisteine attenuates TNFalpha-induced inflammation in human alveolar epithelial cells in vitro through suppressing NF-kappaB and ERK1/2 MAPK signaling pathways. Acta Pharmacol Sin. 2016;37:629-636. doi:10.1038/ aps.2015.150

8. Wang W, Zheng JP, Zhu SX, et al. Carbocisteine attenuates hydrogen peroxide-induced inflammatory injury in A549 cells via NF-kappaB and ERK1/2 MAPK pathways. Int Immunopharmacol. 2015;24:306313. doi:10.1016/j.intimp.2014.12.018

9. Pace E, Di Vincenzo S, Ferraro M, et al. Carbocysteine counteracts the effects of cigarette smoke on cell growth and on the SIRT1/ FoxO3 axis in bronchial epithelial cells. Exp Gerontol. 2016;81:119-128. doi:10.1016/j.exger.2016.05.013

10. Hanaoka M, Droma Y, Chen Y, et al. Carbocisteine protects against emphysema induced by cigarette smoke extract in rats. Chest. 2011;139:1101-1108. doi:10.1378/chest.10-0920

11. Yageta Y, Ishii Y, Morishima Y, et al. Carbocisteine reduces virusinduced pulmonary inflammation in mice exposed to cigarette smoke. Am J Respir Cell Mol Biol. 2014;50:963-973. doi:10.1165/ rcmb.2012-02920C

12. Ishibashi Y, Takayama G, Inouye Y, Taniguchi A. Carbocisteine normalizes the viscous property of mucus through regulation of fucosylated and sialylated sugar chain on airway mucins. Eur $J$ Pharmacol. 2010;641:226-228. doi:10.1016/j.ejphar.2010.05.045

13. Ishibashi Y, Inouye Y, Taniguchi A. [Expression and role of sugar chains on airway mucus during the exacerbation of airway inflammation]. Yakugaku Zasshi. 2012;132:699-704. doi:10.1248/ yakushi.132.699

14. Fahy JV, Dickey BF. Airway mucus function and dysfunction. $N$ Engl $J$ Med. 2010;363:2233-2247. doi:10.1056/NEJMra0910061

15. Thornton DJ, Rousseau K, McGuckin MA. Structure and function of the polymeric mucins in airways mucus. Annu Rev Physiol. 2008;70:459-486. doi:10.1146/annurev.physiol.70.113006.100702

16. Evans CM, Raclawska DS, Ttofali F, et al. The polymeric mucin Muc5ac is required for allergic airway hyperreactivity. Nat Commun. 2015;6:6281. doi:10.1038/ncomms7281

17. Lachowicz-Scroggins ME, Yuan S, Kerr SC, et al. Abnormalities in MUC5AC and MUC5B protein in airway mucus in asthma. Am J Respir Crit Care Med. 2016;194:1296-1299. doi:10.1164/rccm.201603-0526LE

18. Woodruff PG, Modrek B, Choy DF, et al. T-helper type 2-driven inflammation defines major subphenotypes of asthma. Am J Respir Crit Care Med. 2009;180:388-395. doi:10.1164/rccm.200903-0392OC

19. Roy MG, Livraghi-Butrico A, Fletcher AA, et al. Muc5b is required for airway defence. Nature. 2014;505:412-416. doi:10.1038/nature12807

20. Kirkham S, Kolsum U, Rousseau K, et al. MUC5B is the major mucin in the gel phase of sputum in chronic obstructive pulmonary disease. Am J Respir Crit Care Med. 2008;178:1033-1039. doi:10.1164/rccm.200803-3910C

21. Yasuo M, Fujimoto K, Imamura H, et al. L-carbocisteine reduces neutrophil elastase-induced mucin production. Respir Physiol Neurobiol. 2009;167:214-216. doi:10.1016/j.resp.2009.04.016 
22. Shin IS, Shin NR, Park JW, et al. Melatonin attenuates neutrophil inflammation and mucus secretion in cigarette smoke-induced chronic obstructive pulmonary diseases via the suppression of ErkSp1 signaling. J Pineal Res. 2015;58:50-60. doi:10.1111/jpi.12192

23. Shu J, Li D, Ouyang H, et al. Comparison and evaluation of two different methods to establish the cigarette smoke exposure mouse model of COPD. Sci Rep. 2017;7:15454. doi:10.1038/s41598-017-15685-y

24. Atkinson JJ, Holmbeck K, Yamada S, et al. Membrane-type 1 matrix metalloproteinase is required for normal alveolar development. Dev Dyn. 2005;232:1079-1090. doi:10.1002/dvdy.20267

25. Sun L, Tang L, Xu Y, et al. The effect and mechanism of action of carbocysteine on airway bacterial load in rats chronically exposed to cigarette smoke. Respirology. 2010;15:1064-1071. doi:10.1111/ j.1440-1843.2010.01816.x

26. Takeyama K, Dabbagh K, Lee HM, et al. Epidermal growth factor system regulates mucin production in airways. Proc Natl Acad Sci U S A. 1999;96:3081-3086. doi:10.1073/pnas.96.6.3081

27. Warboys CM, Chen N, Zhang QP, et al. Bidirectional cross-regulation between the endothelial nitric oxide synthase and beta-catenin signalling pathways. Cardiovasc Res. 2014;104:116-126. doi:10.1093/cvr/cvu173

28. Fujisawa T, Chang MM, Velichko S, et al. NF-kappaB mediates IL-1betaand IL-17A-induced MUC5B expression in airway epithelial cells. Am J Respir Cell Mol Biol. 2011;45:246-252. doi:10.1165/rcmb.2009-0313OC

29. Allam R, Scherbaum CR, Darisipudi MN, et al. Histones from dying renal cells aggravate kidney injury via TLR2 and TLR4. J Am Soc Nephrol. 2012;23:1375-1388. doi:10.1681/Asn.2011111077

30. Wei Z, Peterson JM, Wong GW. Metabolic regulation by C1q/TNFrelated protein-13 (CTRP13): activation of AMP-activated protein kinase and suppression of fatty acid-induced JNK signaling. $J$ Biol Chem. 2011;286:15652-15665. doi:10.1074/jbc.M110.201087

31. Xue W, Kitzing T, Roessler S, et al. A cluster of cooperating tumorsuppressor gene candidates in chromosomal deletions. Proc Natl Acad Sci USA. 2012;109:8212-8217. doi:10.1073/pnas.1206062109

32. Du RH, Richmond BW, Blackwell TS Jr., et al. Secretory IgA from submucosal glands does not compensate for its airway surface deficiency in chronic obstructive pulmonary disease. Virchows Arch. 2015;467:657-665. doi:10.1007/s00428-015-1854-0

33. Chillappagari S, Preuss J, Licht S, et al. Altered protease and antiprotease balance during a COPD exacerbation contributes to mucus obstruction. Respir Res. 2015;16:85. doi:10.1186/s12931-015-0247-x
34. Rogers DF. Mucus pathophysiology in COPD: differences to asthma, and pharmacotherapy. Monaldi Arch Chest Dis. 2000;55:324-332.

35. Chen Y, Thai P, Zhao YH, et al. Stimulation of airway mucin gene expression by interleukin (IL)-17 through IL-6 paracrine/autocrine loop. J Biol Chem. 2003;278:17036-17043. doi:10.1074/jbc.M210 429200

36. Bracke KR, D'Hulst AI, Maes T, et al. Cigarette smoke-induced pulmonary inflammation and emphysema are attenuated in CCR6deficient mice. J Immunol. 2006;177:4350-4359. doi:10.1111/j.15325415.2007.01373.x

37. Hautamaki RD, Kobayashi DK, Senior RM, Shapiro SD. Requirement for macrophage elastase for cigarette smoke-induced emphysema in mice. Science. 1997;277:2002-2004. doi:10.1126/ science.277.5334.2002

38. Beckett EL, Stevens RL, Jarnicki AG, et al. A new short-term mouse model of chronic obstructive pulmonary disease identifies a role for mast cell tryptase in pathogenesis. $J$ Allergy Clin Immunol. 2013;131:752-762. doi:10.1016/j.jaci.2012.11.053

39. Shu J, Lu W, Li D, et al. [Nose-only cigarette smoke exposure plus airway lipopolysaccharide inhalation induced chronic obstructive pulmonary disease and associated pulmonary hypertension in mice]. Zhonghua Jie He He Hu Xi Za Zhi. 2015;38:907-911.

40. Caramori G, Di Gregorio C, Carlstedt I, et al. Mucin expression in peripheral airways of patients with chronic obstructive pulmonary disease. Histopathology. 2004;45:477-484. doi:10.1111/j.13652559.2004.01952.x

41. Innes AL, Woodruff PG, Ferrando RE, et al. Epithelial mucin stores are increased in the large airways of smokers with airflow obstruction. Chest. 2006;130:1102-1108. doi:10.1378/chest.130.4. 1102

42. O'Donnell RA, Richter A, Ward J, et al. Expression of ErbB receptors and mucins in the airways of long term current smokers. Thorax. 2004;59:1032-1040. doi:10.1136/thx.2004.028043

43. Kubo F, Ariestanti DM, Oki S, et al. Loss of the adhesion G-protein coupled receptor ADGRF5 in mice induces airway inflammation and the expression of CCL2 in lung endothelial cells. Respir Res. 2019;20 (1):11. doi:10.1186/s12931-019-0973-6

\section{Publish your work in this journal}

Drug Design, Development and Therapy is an international, peerreviewed open-access journal that spans the spectrum of drug design and development through to clinical applications. Clinical outcomes, patient safety, and programs for the development and effective, safe, and sustained use of medicines are a feature of the journal, which has also been accepted for indexing on PubMed Central. The manuscript management system is completely online and includes a very quick and fair peer-review system, which is all easy to use. Visit http://www. dovepress.com/testimonials.php to read real quotes from published authors. 